\title{
Poisoning due to consumption of horse chestnut seed
}

\author{
Hwa Yeon $\mathrm{Yi}^{1}$, Jang Young Lee ${ }^{2}$ \\ 'Department of Emergency Medicine, Konyang University Hospital, Daejeon, Korea \\ ${ }^{2}$ Department of Emergency Medicine, Eulji University College of Medicine, Daejeon, Korea
}

Horse chestnut (Aesculus hippocastanum) is a common tree found on roads and parks. The shape of the fruit is very similar to that of the edible Korean chestnut (Castanea crenata); thus, people can eat it by mistake. However, reports of the side effects and toxicity from ingestion are very rare. A 46-year-old male who had no unusual findings in the past had eaten horse chestnut seed which he had mistaken to be Korean chestnut. He visited the emergency department (ED) with complaints of epigastric pain, nausea, and sweating. Blood tests showed a slight increase in the levels of liver enzymes, serum amylase, and pancreatic amylase. During the monitoring, he complained of palpitations, and electrocardiogram showed atrial fibrillation. On the following day after conservative treatment, blood testing and electrocardiogram showed normal findings. He was discharged from the ED as he did not complain of any further symptoms. When a patient who has eaten horse chestnut visits the ED, blood examination and electrocardiogram monitoring are needed, and conservative treatment is required.

Keywords Aesculus; Hippocastanaceae; Poisoning; Atrial fibrillation

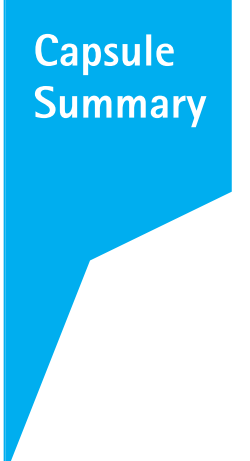

What is already known

The consumption of horse chestnut seeds can lead to serious side effects, including gastrointestinal symptoms, dizziness, headache, and itching.

What is new in the current study

Ingestion of horse chestnut seed manifests not only mild symptoms such as gastrointestinal symptoms but also severe toxicities, including pancreatic toxicity, hepatotoxicity, and cardiac toxicity leading to atrial fibrillation.
eISSN: 2383-4625

Received: 20 August 2020

Revised: 23 September 2020

Accepted: 24 September 2020

Correspondence to: Jang Young Lee Department of Emergency Medicine,

Eulji University Hospital, 95

Dunsanseo-ro, Seo-gu, Daejeon 35233,

Korea

E-mail: pons1224@eulji.ac.kr

ORCID

https://orcid.org/0000-0003-2679-6687

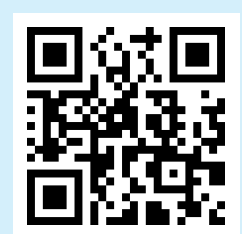

How to cite this article:

Yi HY, Lee JY. Poisoning due to consumption of horse chestnut seed. Clin Exp Emerg Med 2021;8(4):333-335. https://doi.

org/10.15441/ceem.20.004

This is an Open Access article distributed under the terms of the Creative Commons Attribution Non-Commercial License (https:// creativecommons.org/licenses/by-nc/4.0/). 


\section{INTRODUCTION}

Horse chestnut (Aesculus hippocastanum) trees are common in South Korea, and the fallen seeds are ubiquitous on the streets during autumn. Their shape and color are similar to those of edible Korean chestnut (Castanea crenata); hence, people sometimes mistake horse chestnuts for Korean chestnuts. The toxicity of horse chestnut seeds is due to saponins, a mixture of many compounds belonging to the flavonoid group. However, most cases of horse chestnut toxicity are not severe; hence, case reports on horse chestnut toxicity are very rare.' This is the first case report highlighting cardiac, hepatic, and pancreatic toxicity in addition to gastrointestinal symptoms associated with horse chestnut consumption.

\section{CASE REPORT}

A 46-year-old man visited the emergency department (ED) with complaints of abdominal pain, nausea, and sweating. Thirty minutes before his ED visit, the patient had eaten a seed resembling a Korean chestnut ( $C$. crenata). He had no other history that might account for the symptoms. He experienced pain in the upper abdomen accompanied by nausea. Physical examination revealed hyperactive bowel sounds, and tenderness in the upper abdomen without rebound tenderness. Blood pressure, pulse rate, respiratory rate, and body temperature were 128/92 mmHg, 62 beats/ min, 24 breaths/min, and $36.2^{\circ} \mathrm{C}$, respectively. Electrocardiogram showed normal sinus rhythm. Laboratory tests revealed elevated levels of aspartate aminotransferase and alanine aminotransfer- ase at 56/68 IU/L (normal range, 1-39/1-39 IU/L), amylase $159 \mathrm{U} /$ $\mathrm{L}$ (normal range, 20-104 U/L), pancreatic amylase $131 \mathrm{U} / \mathrm{L}$ (normal range, 13-53 U/L), and lactate dehydrogenase of $624 \mathrm{IU} / \mathrm{L}$ (normal range, 240-460 IU/L). These parameters were within normal ranges 2 months earlier, when the patient received an annual health examination. While under observation, the patient suddenly complained of palpitations, and electrocardiogram showed atrial fibrillation with a pulse rate of 132 beats/min (Fig. 1). This symptom disappeared after a few hours of observation, and the pulse rate returned to normal without medical intervention. Normal saline, famotidine $20 \mathrm{mg}$, and metoclopramide $\mathrm{HCl} 10 \mathrm{mg}$ were administered intravenously to treat pain in the upper abdomen accompanied by nausea, and $500 \mathrm{~mL}$ of nafamostat mesylate $10 \mathrm{mg}$ dissolved in 5\% dextrose solution was injected intravenously for 2 hours to treat elevated levels of pancreatic enzymes. The patient was admitted to the ED for overnight observation. The next day, serum aspartate aminotransferase, alanine aminotransferase, and pancreatic amylase levels showed normal results, and the patient was discharged Since his abdominal pain and abnormal symptoms had subsided.

\section{DISCUSSION}

The seeds of horse chestnut (A. hippocastanum) resemble those of Korean chestnut ( $C$. crenata), particularly in shape and color. However, unlike Korean chestnuts, horse chestnuts do not have sharp tips on their seeds, which may be used to distinguish the two seeds (Fig. 2). During autumn, owing to the abundant fallen horse chestnut seeds, people can mistake horse chestnut seeds

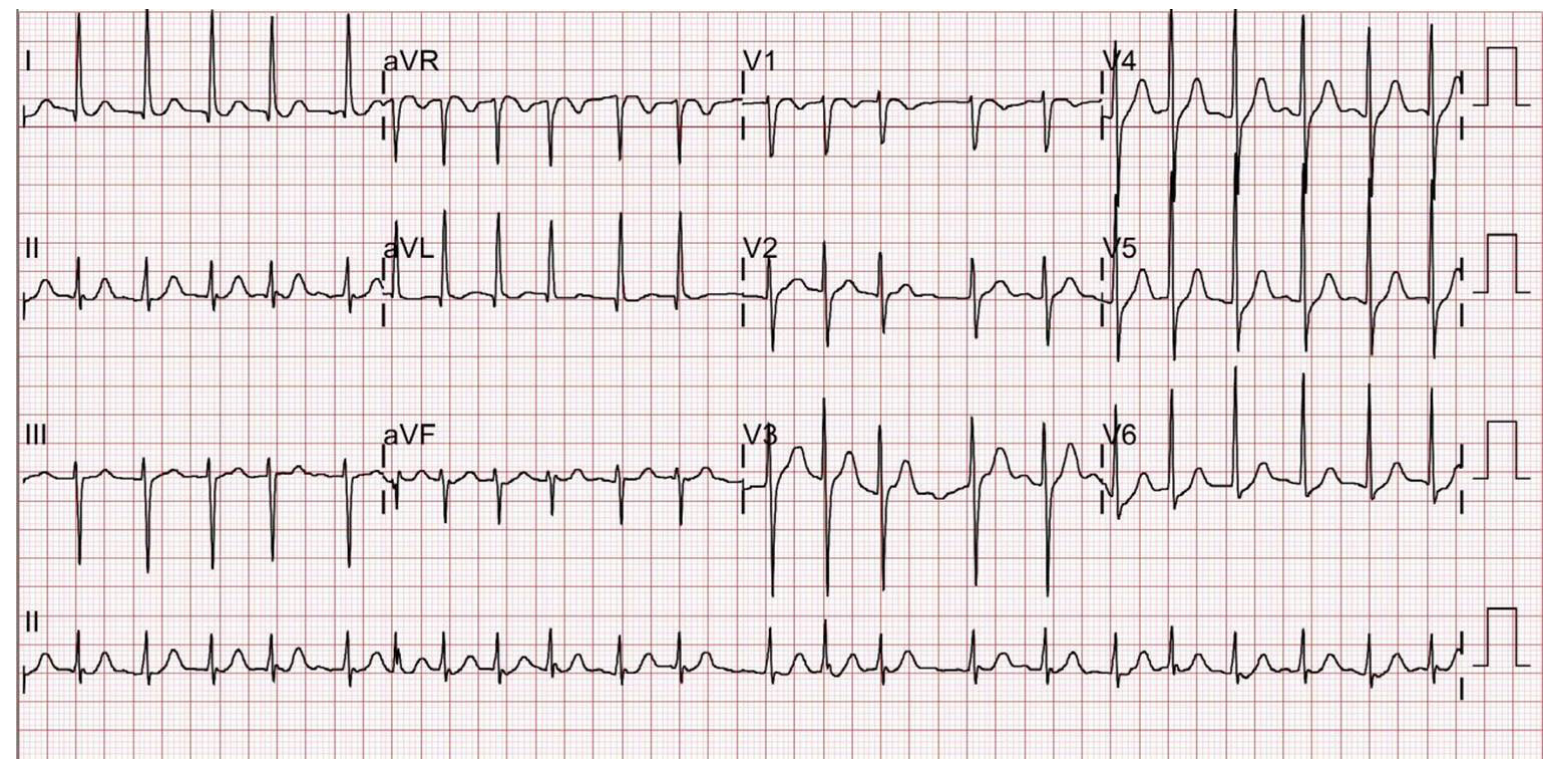

Fig. 1. Electrocardiogram showing atrial fibrillation. 

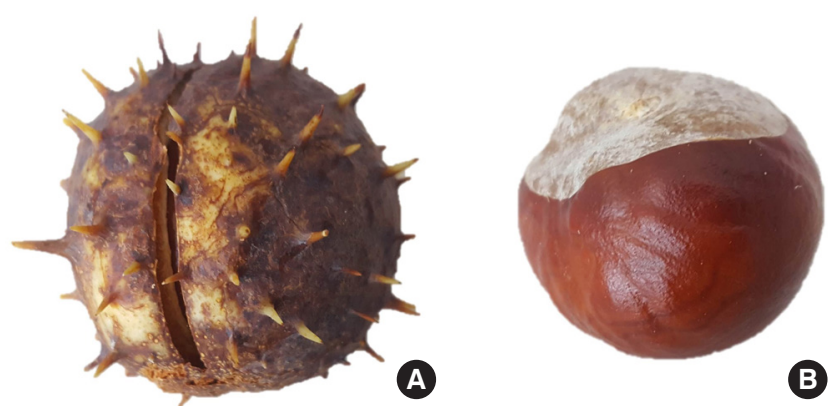

Fig. 2. Horse chestnut fruit (A) and seed (B).

for Korean chestnuts. Because Korean chestnuts can be eaten raw, mistaking horse chestnuts for Korean chestnuts can lead to toxicity when they are inadvertently consumed. ${ }^{2}$

The most active chemical component in horse chestnuts is aescin, comprising two structures: $\alpha$-aescin and $\beta$-aescin of the triterpenoid saponins. ${ }^{3}$ Moreover, they contain bioflavonoids such as quercetin and kaempferol and antioxidants including proanthocyani$\operatorname{din} A 2$, coumarins fraxin, and aesculin. ${ }^{1,2}$ Historically, the seed extract was used for treating ailments including rheumatism, rectal complaints, fever, hemorrhoids, and leg cramps. Currently, horse chestnut seed extract is widely used in Europe for treating chronic venous insufficiency, hemorrhoids, postoperative edema, and topically for various skin conditions. In the United States, horse chestnut seed extract is gaining wider acceptance as an effective therapy for venous disorders and edema, based on results from randomized controlled trials published over the last two decades. ${ }^{1,2}$

However, the ingestion of these fruits can lead to serious side effects. The frequency of adverse effects when using substances containing aescin is $0.5 \%$ to $3 \%$, with the most common symptoms including gastrointestinal disturbances, dizziness, itching, urticaria, and respiratory issues. ${ }^{4}$ Gastrointestinal symptoms usually occur at high doses, and acute anaphylactic reactions have been reported when applied to the skin. ${ }^{1,4}$

A study reported that a patient with a history of allergy who used intranasal powdered horse chestnut seeds had vomiting, dyspnea, burning in the nose and throat, and syncope. ${ }^{5}$ In another case, a 32-year-old man had complaints of dyspnea after consuming three boxes of horse chestnut paste over 1.5 months and was diagnosed with acute exudative pericarditis. ${ }^{6}$ The possible mechanisms underlying the development of pericardial effusion include the strong antiaggregant effects and the production of antibodies as an immunologic response to horse chestnut antigens. ${ }^{6}$ However, in contrast to our case, symptoms in the previous cases did not occur immediately after ingestion, and there were no reports of atrial fibrillation. The severe symptoms in our case may be due to the consumption of an entire unpurified horse chestnut seed; thus, exposing the body to a substantially high amount of antigen(s). ${ }^{1,6}$ The exact mechanism is unclear, but we speculate that the vagus nerve and autonomic nervous system might be implicated in the atrial fibrillation.

Previous studies have reported no abnormalities in the levels of liver enzymes and serum/pancreatic amylase. In our case, the abnormalities observed, although temporary, could have a negative effect on the liver and pancreas.

Ingestion of even a small amount of horse chestnut seed manifests not only mild symptoms such as gastrointestinal symptoms but also severe toxicities, including pancreatic toxicity, hepatotoxicity, and cardiac toxicity, leading to atrial fibrillation. Therefore, electrocardiogram monitoring is essential, and conservative treatment should be performed alongside fluid therapy.

\section{CONFLICT OF INTEREST}

No potential conflict of interest relevant to this article was reported.

\section{REFERENCES}

1. Sirtori CR. Aescin: pharmacology, pharmacokinetics and therapeutic profile. Pharmacol Res 2001;44:183-93.

2. Aesculus hippocastanum (Horse chestnut). Monograph. Altern Med Rev 2009;14:278-83.

3. Wilkinson JA, Brown AM. Horse chestnut: Aesculus hippocastanum: potential applications in cosmetic skin-care products. Int J Cosmet Sci 1999;21:437-47.

4. Escribano MM, Munoz-Bellido FJ, Velazquez E, et al. Contact urticaria due to aescin. Contact Dermatitis 1997;37:233.

5. Zajac M, Wisniewski M, Sein Anand J. Intoxication by powdered seeds of horse chestnut (Aesculus hippocastanum) used nasally as snuff: a case report. Przegl Lek 2014;71:502-3.

6. Edem E, Kahyaoglu B, Cakar MA. Acute effusive pericarditis due to horse chestnut consumption. Am J Case Rep 2016;17: 305-8.

7. Groh CA, Faulkner M, Getabecha S, et al. Patient-reported triggers of paroxysmal atrial fibrillation. Heart Rhythm 2019;16: 996-1002. 